\title{
Violent Sabotage of Mass-Gathering Events
}

\author{
Samuel J. Stratton, MD, MPH
}

Research and evaluation of health and medical response during mass-gathering events originated during the early phases of disaster medical science. While there is no standard definition of a mass gathering, a simple way of conceptualizing a mass gathering is as an event with a theme occurs at a location and more people than usual for the location come together. Because mass gatherings occur as a result of a common theme or objective, there is a tendency for groups of people with similar ideas and interests to attend. This gathering of people with similar interests or beliefs allows for identification of a group that can be targeted for sabotage and injury by those who disagree or are angered by the event or people who attend.

While spontaneous and unplanned mass gatherings occur, there is usually pre-warning of the event that allows for planning of health and medical response needs. Extensive work has been published with a focus on the best methods for such planning and response. ${ }^{1}$ Factors such as weather and ambient temperature, location and event theme, food and alcohol availability, and access to water for hydration are all important in planning the medical response for mass gatherings.

An emerging challenge for mass-gathering health and medical preparation is the threat of violent sabotage during an event. While terrorism should be considered a form of sabotage, the term "terrorism" is not broad enough to include all the facets that should be considered when preparing for potential violent disruption of a mass gathering. Terrorism implies a systematic use of emotional or physical violence to achieve a recognized end. On the other hand, the broader term "sabotage" includes violent and intimidating acts that lack systematic characteristics and have poorly-defined end goals.

Recognizing the diversity of potential sources for violence at mass gatherings is important. Planners and responders should recognize that some of those sabotaging an event may act unpredictably and with less rational behavior than what is expected of traditional terrorists. This is best illustrated by recent bombings during the US Boston Marathon in which there appears to have been little or no true objective and a minimum of logical behavior. The lack of logic or objective makes anticipating and planning for such acts difficult for both mass-gathering security and medical personnel.

Risk assessment for violence occurring during a mass gathering is difficult and methods of violence are not easy to predict. While explosive devices seem to be favored by many, there is also potential for toxic gas releases (such as Sarin) and poisoning of available food or water. These and other sabotage methods are readily available to those with an interest in violent disruption of mass gatherings.

Health preparation and medical response for mass gatherings is one of the more developed areas in disaster medical science. ${ }^{2}$ Predicting the number of providers needed, and their level of expertise, to give expected medical care during mass-gathering events has become sophisticated and is based upon robust science and evidence. But with an increasing tendency for sabotage, there is a need to redirect some mass-gathering medical research toward this newer threat.

Particularly important is research that provides evidence to allow contingency planning for types of violent acts. Along with recognizing threats, there is a need to better define the proper response by medical responders, as well as responder safety, medical management, and evacuation of casualties. Perfecting syndromic surveillance techniques relative to mass-gathering events is also an area for further research. Prehospital and Disaster Medicine encourages researchers to explore and submit work related to the new challenges for improving health and safety during mass gatherings.

\section{References}

1. Molloy MS, Sherif Z, Natin S, McDonnell J. Management of Mass Gatherings. In: Koenig KL, Schultz CH, eds. Disaster Medicine: Comprehensive Principles and Practice. Cambridge, UK: Cambridge University Press; 2010:228-252.

2. Arbon P. Mass-gathering medicine: a review of the evidence and future directions for research. Prehosp Disaster Med. 2007;22(2):131-135. 